
\title{
25 Research Soure \\ Bioinformatics Analysis Predicts ceRNA Regulatory Axes Related to Melanoma Pathogenesis
}

Lina Liu ( $\sim 1072662286 @ q q . c o m$ )

the second affiliated hospital of hengyang medical school,University of south China

Feng Yang

the second affiliated hospital of hengyang medical school,University of south China

Saiyun Lei

the second affiliated hospital of hengyang medical school,University of south China

\section{Research Article}

Keywords: Bioinformatics Analysis, ceRNA, Skin cutaneous melanoma, circRNA, EMT

Posted Date: September 17th, 2021

DOI: https://doi.org/10.21203/rs.3.rs-871271/v1

License: (1) This work is licensed under a Creative Commons Attribution 4.0 International License.

Read Full License 


\section{Abstract}

Background Melanoma is a highly malignant skin tumour, with an incidence and mortality rates accounting for approximately $5 \%$ and $>75 \%$ of all tumours, respectively. In the present study, we aimed to screen mRNAs, microRNAs, and circRNAs related to the pathogenesis of melanoma via bioinformatics methods.

Materials and methods The microarray data correlating with melanoma were obtained from the GEO database, and the differentially expressed genes between melanomatissues and non-melanoma tissues were screened using GEO2R. Moreover, the Hub genes were screened using the STRING database, Cytoscape software, and GEPIA database. The DAVID database was used for gene enrichment analysis. Thereafter, the ceRNA network diagram was constructed using the starBase database and was analysed for survival in order to predict the molecular markers for diagnosis and treatment of skin cutaneous melanoma; the most probable ceRNA mechanism was screened via pan-cancer analysis. Furthermore, the ceRNA network was verified using the GEPIA and UALCAN databases.

Results In total, 266, 20, and 10 differentially expressed mRNAs, microRNAs, and circRNA10 were screened. Using Cytoscape software, 59 Hub and 31 key genes were screened, followed by construction of the ceRNA and ceRNA-gene enrichment analysis networks.

Conclusion Clinical verification revealed that SIPA1L1 could regulate the expression of DSC3 by sponge adsorption of has-miR-106b and has-miR-20b, and thereby affect the pathogenesis and progression of skin cutaneous melanoma.

\section{Background}

Melanoma is a highly malignant skin tumour, with the incidence and mortality rates accounting for approximately $5 \%$ and $>75 \%$ among all skin tumours. After metastasisation, the skin cutaneous melanoma (SKCM) progresses rapidly with a poor prognosis. At present, the early diagnosis of SKCM mainly depends on dermoscopy and pathology. As the pathological structures of SKCM vary remarkably, certain non-characteristic changes pose difficulties for clinicians in diagnosing this disease. Certain issues persist in melanoma treatment in addition to the diagnosis defects. At present, the treatment methods for melanoma mainly include surgery, radiotherapy, chemotherapy, and immunotherapy. Immunotherapy has proven to be beneficial in SKCM treatment; however, it presents disadvantages such as limited treatment scope.

Competitive endogenous RNA mechanism is an important mechanism of endogenous RNA interaction, wherein the endogenous competitive RNA competes for the binding site of messenger RNA by complementary pairing with specific microRNA through its response elements, thereby resulting in altered mRNA expression level. The three protagonists in this process are ceRNA, miRNA, and mRNA. CeRNAs include long-chain non-coding RNA (IncRNA) and cyclic RNA. The circRNA differs from IncRNA, as it has a closed-loop stable structure and conservative sequence; therefore, it cannot be easily degraded by 
various enzymes in the cells, and can be identified as a stably expressed biomarker, thereby exhibiting unique advantages in disease diagnosis and treatment.

In the present study, the melanoma data was screened, analysed, and visualised via bioinformatics analysis, followed by construction of the ceRNA network related to the pathogenesis of melanoma. In addition, the ceRNA regulatory axes in the network were verified, and finally the most likely ceRNA regulatory axis was predicted. Identification of specific biomarkers for early diagnosis of melanoma and effective treatment methods and targets has become a breakthrough in melanoma treatment.

\section{Results}

\subsection{Screening of differentially expressed genes}

Volcanic maps of gene expression were plotted for the mRNA datasets GES114445 and GSE7553, and the microRNA datasets GSE18512, GSE35579, and GSE24996, respectively (Figs. 1A-E). Data were analysed using GEO2R with screening conditions at values $p<0.05$ and $|\log \mathrm{FC}|>1$. In total, the GES114445 data set comprised 839 differentially expressed genes, including 528 up-regulated and 311 down-regulated genes. Moreover, the GSE7553 data set consisted of 1776 differentially expressed genes, including 551 upregulated and 1225 down-regulated genes. Furthermore, the GSE18512 data set comprised 22 differentially expressed genes, including 14 up-regulated and 8 down-regulated genes. Additionally, the GSE35579 data set consisted of 52 differentially expressed genes, including 18 upregulated and 34 down-regulated genes. Eventually, the GSE24996 data set comprised 48 differentially expressed genes, including 20 up-regulated and 28 down-regulated genes.

An article entitled "Epigenetic silencing of CDR1 as drives IGF2BP3-mediated melanoma invasion and metastasis" was retrieved from PubMed, and the circRNA differential expression data (the GSE138711 chip set from the GEO database) were downloaded. In total, 688 differentially expressed circRNAs were identified, including 572 differentially down-regulated and 116 up-regulated genes. The 10 most differentially expressed circRNAs were screened as the target molecules for subsequent research. Among these, the up-regulated circRNAs were LIFR, ZC3H13, ARID1A, XRN2, and ITCH, whereas the downregulated circRNAs were SIPA1L1, WDR37, PAG1, ARHGEF12, and CCSER2 (Fig. 2, Table 1).

The intersection of the above-mentioned differential genes was obtained by drawing Venn diagrams of 283 and 26 common differentially expressed mRNAs and microRNAs, respectively (Figs. 3A, B).

Venn of differentially expressed gene intersection. A the intersection of GSE114445 and GSE7553. B the intersection of GSE18512 GSE35579 and GSE24996. (the genes contained in at least two data sets were selected as the target genes)

\subsection{Acquisition of Hub genes}

A network of 283 differentially expressed mRNAs was constructed in the STRING database to obtain a PPI network with 184 nodes and 490 edges; thereafter, the PPI network file was downloaded. Excel was 
used to collate the data in the network file, and two files 'Node to Node Network' and 'degree', were obtained. These files were imported into Cytoscape software and the network was enhanced, thereby obtaining the PPI network diagram as illustrated in Fig. 4. Moreover, 59 genes with degree value $>$ mean degree value were screened as the Hub genes, and a sub-network was established (Fig. 5).

The subnetwork of Hub genes. Icons with different colors represent the degree value of the gene. The ligther the color, the smaller the degree value of the gene; Different icons represent the expression trend of the gene, the triangle the down-regulation of the gene expression in skcm, and the arrow symbol represents the up-regulation of the gene expression in skcm; The letters on the icon represest the name of the gene.

\subsection{GO and KEGG enrichment analysis of Hub genes}

The GO and KEGG enrichment analyses were performed for 59 Hub genes in the DAVID database with screening conditions of $p<0.05$ and count value $>$ mean count value. Biological effects were mainly enriched in inflammatory response, immune response, innate immune response, positive regulation of ERK1 and ERK2, and drug response. Molecular functions were mainly enriched in protein binding. Moreover, cell localisation was mainly enriched in plasma membrane, extracellular exosomes, components of plasma membrane, cell surface, and desmosomes. Furthermore, the signalling pathways were mainly enriched in Staphylococcus aureus infection, osteoclast differentiation, cytotoxicity mediated by natural killer cells, influenza $A$, and in the chemokine signalling pathway. Figure $6 \mathrm{~A}$ and $6 \mathrm{~B}$ illustrate the results of GO analysis with more than three genes and the KEGG pathway enrichment analysis, respectively.

The results of $\mathrm{GO}$ and KEGG enrichment analysis. A the results of $\mathrm{GO}$ enrichment analysis(The GO_BP,GO_MF and GO_CC were selected with thresholds of $\mathrm{P}<0.05$ and count $>3$ ); $\mathrm{B}$ the results of KEGG enrichment analysis $\square$ The KEGG signaling pathways were selected with threshold of $P<0.05 \square$

\subsection{Survival analysis for Hub genes and identification of key genes}

In order to screen the Hub genes and clarify their significance in melanoma, the 59 Hub genes screened above were imported into the GEPIA database for survival analysis $(P<0.05)$. In total, 31 key genes correlating with the overall survival rate of melanoma were screened, among which 25 genes were differentially up-regulated and 6 genes were differentially down-regulated (Table 1, Figs. 7). 
Table 1

Correlation between mRNA and survival rate of SKCM

\begin{tabular}{|lll|}
\hline gene name & variation trend & P value \\
\hline FBXW7 & Down & $3.30 \mathrm{E}-02$ \\
\hline DSG1 & Down & $9.30 \mathrm{E}-03$ \\
\hline EVPL & Down & $2.60 \mathrm{E}-05$ \\
\hline PKP1 & Down & $3.50 \mathrm{E}-04$ \\
\hline DSC3 & Down & $6.50 \mathrm{E}-03$ \\
\hline EPPK1 & Down & $6.10 \mathrm{E}-03$ \\
\hline CXCR4 & Up & $2.10 \mathrm{E}-02$ \\
\hline AURKA & Up & $1.40 \mathrm{E}-02$ \\
\hline CYBB & Up & $3.40 \mathrm{E}-05$ \\
\hline CCR1 & Up & $5.70 \mathrm{E}-05$ \\
\hline CCL5 & Up & $4.50 \mathrm{E}-06$ \\
\hline ICAM1 & Up & $2.60 \mathrm{E}-04$ \\
\hline CD86 & Up & $1.40 \mathrm{E}-06$ \\
\hline LCP2 & Up & $2.00 \mathrm{E}-06$ \\
\hline TLR7 & Up & $4.00 \mathrm{E}-04$ \\
\hline TYR0BP & Up & $1.30 \mathrm{E}-03$ \\
\hline FCGR2A & Up & $4.30 \mathrm{E}-06$ \\
\hline C1QC & Up & $9.30 \mathrm{E}-06$ \\
\hline CD53 & Up & $5.00 \mathrm{E}-05$ \\
\hline FCER1G & Up & $9.90 \mathrm{E}-05$ \\
\hline KPNA2 & Up & $1.80 \mathrm{E}-03$ \\
\hline LAPTM5 & Up & $1.50 \mathrm{E}-06$ \\
\hline DTL & Up & $1.00 \mathrm{E}-03$ \\
\hline FPR3 & Up & \\
\hline FYB & Up & \\
\hline NCF1 & Up & \\
\hline
\end{tabular}




\begin{tabular}{|lll|}
\hline gene name & variation trend & P value \\
\hline SERPINA1 & Up & $2.90 \mathrm{E}-04$ \\
\hline HAVCR2 & Up & $8.70 \mathrm{E}-06$ \\
SRGN & Up & $8.20 \mathrm{E}-07$ \\
FCGR1B & Up & $8.70 \mathrm{E}-06$ \\
\hline GBP5 & Up & $3.20 \mathrm{E}-07$ \\
\hline
\end{tabular}

Correlation between mRNA and survival rate of SKCM. The mRNAs were selected with threshold of Logrank $\mathrm{P}<0.05$

\subsection{Construction of the ceRNA network}

The miRNA-mRNA column in the starBase database was used to predict the corresponding relationships between the miRNAs and key genes, and the co-expression analysis of miRNAs and their corresponding mRNAs was performed in the starBase database. Based on the contradictory changing trends of miRNAs and mRNAs in the ceRNA network, the screening condition (coefficient-R $<0$ ) was set for filtration, and the microRNA-mRNA docking relationships were obtained (Table 2).

In the miRNA-circRNA column of starBase database, the corresponding relationships of miRNAs with circRNA LIFR, ZC3H13, ARID1A, XRN2, ITCH, SIPA1L1, WDR37, PAG1, ARHGEF12, and CCSER2, were predicted. As circRNA often acts as a miRNA sponge in the ceRNA network to competitively inhibit the binding of miRNAs and mRNAs, the expression trends of circRNAs in the circRNA-miRNA-mRNA relationship are consistent with those of mRNAs, but contradictory to those of miRNAs. Based on the above-mentioned principles, we screened the following miRNA-circRNA corresponding relationships (Table 3). The miRNA-mRNA pairing relationships were integrated with the miRNA-circRNA pairing relationships, thereby obtaining the ceRNA network diagram (Fig. 8). 
Table 2

Correlation between mRNA and miRNA

\begin{tabular}{|c|c|c|c|c|}
\hline mRNA & Variation trend & microRNA & Coeffient-R & $P$ value \\
\hline \multirow[t]{5}{*}{ FBXW7 } & Down & Hsa-miR-155 & -0.071 & 1.31E-01 \\
\hline & & Hsa-miR-21 & -0.156 & 8.90E-04 \\
\hline & & Hsa-miR-196a & -0.196 & 2.77E-05 \\
\hline & & Hsa-miR-425 & -0.225 & 1.53E-06 \\
\hline & & Hsa-miR-142 & -0.019 & 6.89E-01 \\
\hline \multirow[t]{2}{*}{ DSG1 } & down & Hsa-miR-130b & -0.118 & $1.26 \mathrm{E}-02$ \\
\hline & & Hsa-miR-142 & -0.054 & 2.58E-01 \\
\hline \multirow[t]{4}{*}{ PKP1 } & down & Hsa-miR-155 & -0.017 & 7.23E-01 \\
\hline & & Hsa-miR-424 & -0.078 & $9.92 \mathrm{E}-02$ \\
\hline & & Hsa-miR-185 & -0.043 & 3.60E-01 \\
\hline & & Hsa-miR-425 & -0.024 & $6.14 \mathrm{E}-01$ \\
\hline \multirow[t]{5}{*}{ DSC3 } & down & Hsa-miR-106b & -0.107 & $2.35 \mathrm{E}-02$ \\
\hline & & Hsa-miR-130b & -0.107 & $2.36 \mathrm{E}-02$ \\
\hline & & Hsa-miR-155 & -0.041 & $3.88 \mathrm{E}-01$ \\
\hline & & Hsa-miR-20b & -0.065 & 1.67E-01 \\
\hline & & Hsa-miR-142 & -0.011 & 8.20E-01 \\
\hline \multirow[t]{3}{*}{ EPPK1 } & down & Hsa-miR-425 & -0.132 & $5.11 \mathrm{E}-03$ \\
\hline & & Hsa-miR-424 & -0.105 & 2.62E-02 \\
\hline & & Hsa-miR-185 & -0.095 & 4.38E-02 \\
\hline \multirow[t]{3}{*}{ ICAM1 } & Up & Hsa-miR-214 & -0.095 & 4.35E-02 \\
\hline & & Hsa-miR-125b & -0.057 & 2.30E-01 \\
\hline & & Hsa-miR-125a & -0.087 & $6.56 \mathrm{E}-02$ \\
\hline LAPTM5 & Up & Hsa-miR-185 & -0.061 & $1.95 \mathrm{E}-01$ \\
\hline \multirow[t]{2}{*}{ DTL } & Up & Hsa-miR-204 & -0.104 & 2.70E-02 \\
\hline & & Hsa-miR-214 & -0.021 & $6.50 \mathrm{E}-01$ \\
\hline \multirow[t]{2}{*}{ AURKA } & Up & Hsa-miR-23b & -0.034 & 4.68E-01 \\
\hline & & Hsa-Let-7b & -0.115 & 1.44E-02 \\
\hline
\end{tabular}




\begin{tabular}{|lllll|}
\hline mRNA & Variation trend & microRNA & Coeffient-R & P value \\
\hline CXCR4 & up & Hsa-miR-211 & -0.238 & $3.39 \mathrm{E}-07$ \\
\hline CYBB & up & Hsa-miR-196b & -0.110 & $2.02 \mathrm{E}-02$ \\
\hline FAPTM5 & up & Hsa-miR-27b & -0.042 & $3.79 \mathrm{E}-01$ \\
\hline
\end{tabular}

ceRNA Network Diagram. This figure clearly expressed the interaction among differentially expressed circRNA, microRNA and mRNA. The rectangular icon on the left represents differentially expressed mRNA; the oval icon on the right represesnts differentially expressed circRNA; the diamond icon in the middle represesnts the expressed microRNA. The color of the icon represents the expression trend of the gene in melanoma; Red is up-regulated and green is down-regulated. 
Table 3

miRNA-circRNA docking relationship

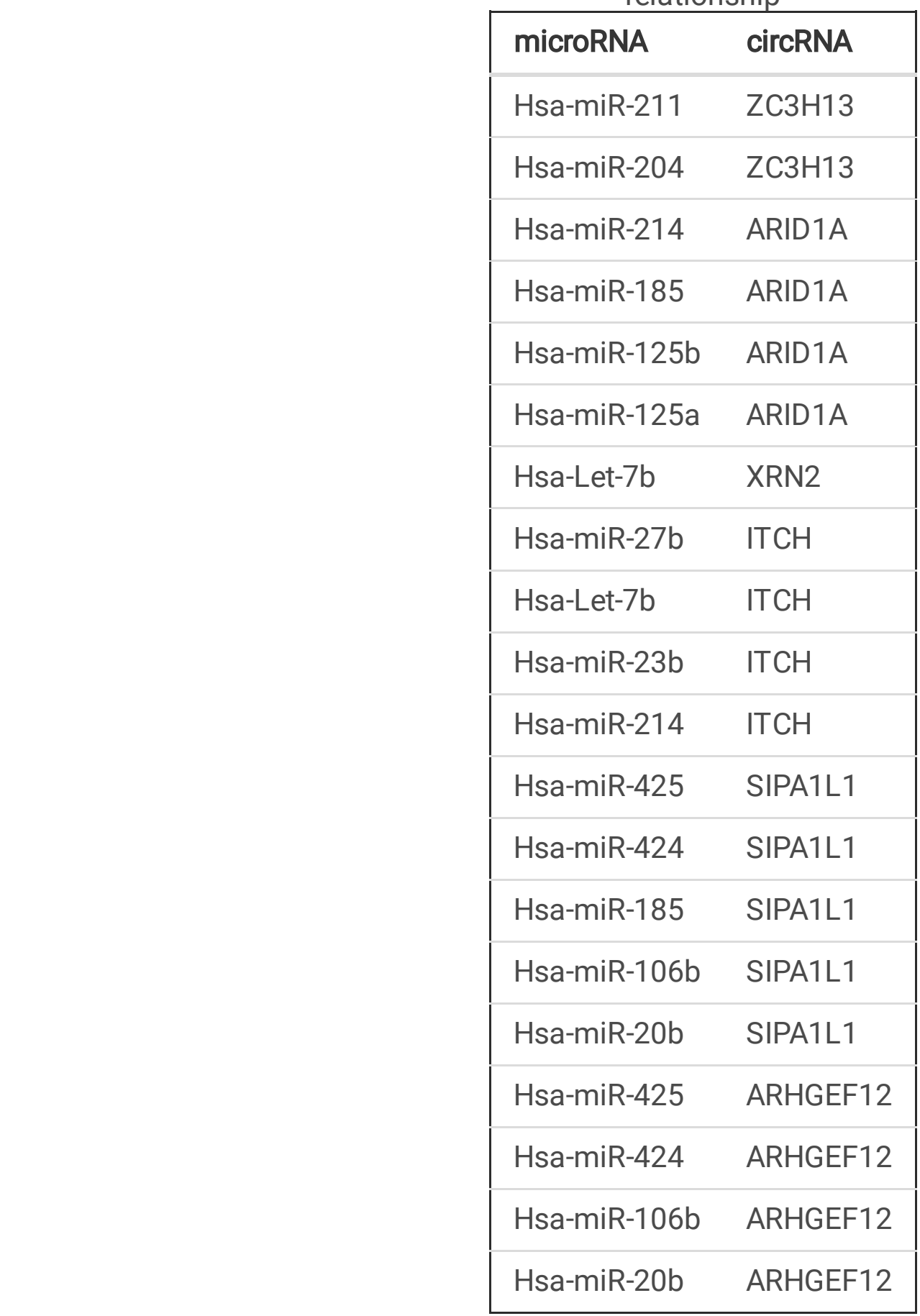

\section{6ceRNA network verification}

The results of mRNA and miRNA co-expression in the ceRNA network were retrieved from the starBase database. The conditions for ceRNA network verification were as follows: (1) $P<0.05$ and (2) at least two common miRNAs between the mRNAs and circRNAs; satisfying one of the above conditions was necessary. The following target ceRNA corresponding relationships were screened based on the above conditions (Table 4). 
The target ceRNAs and their corresponding GO/KEGG enrichment analysis results were plotted in Cytoscape (Fig. 9).

Differential analysis of mRNAs from the target ceRNA network was carried out in the GEPIA database, and the pan-cancer expression patterns obtained are illustrated in Fig. 10. Compared with other tumours, the expression of DSC3, PKP1, and EPPK1 in melanoma and normal skin tissue was specific. The correlation between target mRNA in the ceRNA network and the staging of melanoma was analysed in the GEPIA database (Fig. 11). Among FBXW7 $(P=0.0402)$, PKP1 $(P=0.000201)$, DSC3 $(P=0.000175)$, EPPK1 $(P=0.0243)$, ICAM $(P=0.423)$, DTL $(P=0.102)$, AURKA $(P=0.0534)$, and CXCR4 $(P=4.94 \mathrm{e}-06)$, only FBXW7, PKP1, DSC3, EPPK1, and CXCR4 were correlated with the tumour staging in melanoma $(P<$ 0.05).

Survival analysis of miRNAs in the target ceRNA network was carried out in the UALCAN database (Fig. 12). Among has-miR-106b ( $P=0.049)$, has-miR-20b $(P=0.027)$, has-miR-185 $(P=0.65)$, has-miR-214 $(P=$ 0.84), has-miR-125b ( $P=0.086)$, has-miR-204 $(P=0.056)$, has-miR-211 $(P=0.19)$, has-miR-425 $(P=0.60)$, has-miR-424 ( $P=0.79)$, and has-let-7b $(P=1)$, only has-miR-106b and has-miR-20b were correlated with the overall survival rate of melanoma $(P<0.05)$.

Eventually, we screened the SIPA1L1-has-miR-106b/has-miR-20b-DSC3 regulatory axis and presumed that SIPA1L1 can regulate DSC3 expression by competitively binding to HAS-miR-106b and HAS-miR-20b, thereby affecting the development and progression of melanoma.

Table 4

mRNA-miRNA-circRNA docking relationship

\begin{tabular}{|clll|}
\hline ㅁ & circRNA & miRNA & mRNA \\
\hline 1 & SIPA1L1 & hsa-miR-425 & FBXW7 \\
2 & SIPA1L1 & Hsa-miR-424/hsa-miR-425 & PKP1 \\
\hline 3 & SIPA1L1 & Hsa-miR-106b/hsa-miR-20b & DSC3 \\
\hline 4 & SIPA1L1 & Hsa-miR-425/hsa-miR-424 & EPPK1 \\
\hline 5 & ARID1A & Hsa-miR-214/hsa-miR-125b/hsa-miR-125a & ICAM1 \\
6 & ZC3H13 & Hsa-miR-214 & DTL \\
\hline 7 & XRN2 & Hsa-let-7b & AURKA \\
8 & ZC3H13 & Hsa-miR-211 & CXCR4 \\
\hline
\end{tabular}

Survival analysis of miRNA in skin cutaneous melanoma. It can be seen from the figure that has-miR$106 \mathrm{~b}$ and has-miR-20b have related to the survival rate of skin cutaneous melanoma(The miRNAs were selected with threshold of $\mathrm{P}<0.05$ ).

\section{Discussion}


Melanoma is a highly malignant skin tumour. Once metastasis occurs, it progresses rapidly and leads to a high mortality rate. Due to the limitations of the present diagnosis technology and treatment methods, some cases of melanoma with atypical changes were misdiagnosed or undiagnosed; moreover, even diagnosed melanoma cases could not achieve the best treatment outcome. Therefore, identifying stable and specific biomarkers as well as effective treatment methods is necessary for treating SKCM.

circleRNA (circRNA) is a special non-coding RNA molecule different from long intergenic noncoding RNA (lincRNA). It is in a closed loop and remains unaffected by RNA exonuclease, with conservative sequence and stable expression. Simultaneously, circRNA absorbs microRNA with its unique MER sponge and affects the expression level of mRNA; thus, it can effectively control the biological process of cells. Therefore, it is of great significance to study the mechanism of circRNA-related ceRNA in the development and progression of tumour diseases.

In the present study, bioinformatics analysis methods were used to predict the ceRNA network correlated with melanoma by means of data mining, data analysis, and data visualisation. The network comprised 6 circRNAs, 19 microRNAs, and 12 mRNAs. Based on the principle of ceRNA mechanism, that is, the expression trends of circRNAs and mRNAs are consistent, whereas that of microRNAs is contradictory to that of circRNAs and mRNAs. Furthermore, a ceRNA sub-network (Fig. 9, Table 4) was screened, comprising 3 circRNAs, 11 microRNAs, and 8 mRNAs. For clinical verification of the network, we analysed the expression characteristics of mRNAs in the ceRNA network in 33 types of tumour tissues and their surrounding normal tissues in the GEPIA database, and found that the expression of DSC3, EPPK, and PKP1 in SKCM was specific and high compared with that of squamous cell carcinoma of the head and neck, presenting low or even no expression in the tumour tissues, but significantly high expression in normal tissues. Therefore, we predicted that DSC3, EPPK, and PKP1 can be used as biomarkers for early diagnosis of melanoma. Moreover, we analysed the differences in expression of these mRNAs at various stages of melanoma in the GEPIA database, where insignificant differences were observed in the expression of FBXW7, PKP1, DSC3, EPPK1, and CXCR4 $(P<0.05)$. Eventually, 11 microRNAs were validated in the UALCAN database. We found that the expression levels of has-miR-106b and has-miR$20 \mathrm{~b}$ were correlated with the overall survival rate of SKCM $(P<0.05)$, and the overall survival rate of patients with high expression of has-miR-106b and has-miR-20b was relatively low. Thus, the ceRNA regulatory axis circ SIPA1L1/has-miR-106b or 20b/DSC3 was screened.

Signal-induced proliferation-associated 1-like protein 1 (SIPA1L1), can form circSIPA1L1 through selfcleavage and cyclisation, and exhibits specific high expression in various tumour tissues. For example, circSIPA1L1 can regulate the expression of MAPK1 through sponge adsorption of has-miR-379-5p and promote the invasion and metastasis of osteosarcoma. Furthermore, circSIPA1L1 can promote proliferation, invasion, and migration of renal cancer cells. The down-regulation of circSIPA1L1 in tumour tissues is rare. In the present study, the expression of circRNA SIPA1L1 in melanoma tissues was downregulated, indicating that circSIPA1L1 has very specific expression in melanoma tissues and thus can serve as an important marker for the diagnosis of melanoma. Has-miR-106b and has-miR-20b reveal higher expression in various tumour tissues. Dr. Drobna et al. reported that has-miR-20b can induce the 
development of lymphocytic leukaemia by affecting the expression of tumour suppressor genes PTEN and BIM. Li et al. found that miR-106b could affect the development and progression of breast cancer by inhibiting the expression of PTEN through the PI3K/AKT pathway. DSC3 is an epithelial-specific expression gene that encodes a calcium-dependent glycoprotein, which is a member of the superfamily of cadherin necessary for cell adhesion and desmosome formation, and is considered to be an important biomarker for certain cancers. E-cadherin is the main cadherin located in epithelial cells. The loss of DSC3 leads to reduced E-cadherin expression in epithelial cells, lack of cell adhesion, and increase in cell motility, which may further induce epithelial-mesenchymal transition (EMT) of tumour cells through multiple signalling pathways (Fig. 13). Thus, E-cadherin plays a pivotal role in the development and progression of tumour diseases. Jiangli Chen et al. found that the expression of DSC3 in colorectal cancer was down-regulated, and DSC3 inhibited the EMT process of tumour tissues by inhibiting the $\mathrm{PI} 3 \mathrm{~K} / \mathrm{AKT}$ signalling pathway and regulating E-cadherin.

Cell adhesion signaling pathway. E-cadherin is the main cadherin located in epithelial cells. The loss of DSC3 leads to reduced E-cadherin expression in epithelial cells, lack of cell adhesion, and increase in cell motility, which may further induce epithelial-mesenchymal transition (EMT) of tumour cells through multiple signalling pathways.

\section{Conclusion}

circSIPA1L1 is highly expressed in the normal epithelial tissues; sponge adsorption of mi-106b and miR$20 \mathrm{~b}$ can regulate the increase in DSC3 expression, promote the expression of cadherin in epithelial cells, lead to increased intercellular adhesion, and control the proliferation, division, and migration of cells. Nevertheless, circSIPA1L1 reveals low specific expression in melanoma, which leads to the abnormal expression of cadherin through the ceRNA regulatory axis, thereby resulting in reduced intercellular adhesion, accelerated proliferation, and increased migration trend.

\section{Materials And Methods}

\subsection{Analysis of differentially expressed genes}

Using "melanoma" as the key word, we searched the GEO database for melanoma-related datasets and screened six datasets, namely, GES114445, GSE7553, GSE18512, GSE35579, GSE24996, and GSE138711. Among these, GES114445 and GSE7553 belong to mRNA datasets; GSE18512, GSE35579, and GSE24996 belong to microRNA datasets; and GSE138711 belongs to circRNA datasets. GEO2R was used to analyse the GES114445, GSE7553, GSE18512, GSE35579, and SE24996 datasets, and the differentially expressed genes were screened using standardised $P$ value $<0.05$ and $|\log F C|>1$. Thereafter, the differentially expressed genes in the mRNA data sets were intersected to obtain the common differentially expressed mRNAs in the Venn Diagram software, the miRNA data sets were intersected in pairs to obtain common differentially expressed miRNAs, and a volcano map was plotted using Sangerbox software. 


\subsection{Acquisition of Hub genes}

The interactions of differentially expressed mRNAs were assessed using STRING software, and the network file was downloaded. PPI network analysis was performed in Cytoscape software, and the genes conforming to degree > mean degree were screened, and the PPI network diagram was constructed.

\subsection{Gene enrichment analysis and visualisation}

The Hub genes were added into the DAVID database to obtain the results of GO functional analysis and KEGG signalling pathway enrichment analysis. The screening conditions were $P<0.05$ and count $>$ mean count. Sangerbox software was used to plot the bubble chart and pie chart, and Cytoscape software was used to plot the protein-biological function/signalling pathway network diagram.

\subsection{Survival analysis of Hub genes}

The GEPIA database was used to perform survival analysis on the Hub genes, and genes correlated with the overall survival rate of melanoma were filtered at a screening condition of $P<0.05$. The corresponding survival analysis charts of these genes were saved.

\subsection{Establishment of ceRNA network relationships}

The Hub gene-microRNA and circRNAs corresponding to the miRNAs were assessed in the sub-column 'miRNA-mRNA' and 'miRNA-circRNA' of the miRNA-Target column in the starBase database, respectively; moreover, screening was performed according to the reverse change trend of mRNAs and microRNAs and that of circRNAs and miRNAs in the ceRNA network to obtain the Hub gene-microRNA and miRNAcircRNA pairing relationships, respectively.

The mRNA-miRNA and circRNA-miRNA corresponding relationship files were established, and the ceRNA network relationship diagram was constructed in Cytoscape software.

\subsection{Validation of the ceRNA network}

Genes in the ceRNA network were validated in the GEPIA and UALCAN databases, respectively. First, the expression status of key genes in different tumours and their surrounding normal tissues was evaluated in the GEPIA database to screen the key genes that are specifically expressed in melanoma. Second, the expression of key genes at different stages of melanoma was analysed in the GEPIA database to screen the key genes correlated with tumour staging. Third, miRNAs correlating with the overall survival rate of melanoma were selected from UALCAN, and related pictures were downloaded.

\section{Abbreviation List}




\begin{tabular}{|ll|}
\hline Full name & abreviation \\
\hline Skin cutaneous melanoma & SKCM \\
\hline Messenger RNA & mRNA \\
\hline Micro RNA & miRNA \\
\hline Circle RNA & circRNA \\
\hline Epithelial-mesenchymal transformation & EMT \\
\hline long-chain non-coding RNA & IncRNA \\
\hline Competitive endogenous RNA & ceRNA \\
\hline Protein to protein interactions & PPI \\
\hline Kyoto Encyclopedia Genes and Genomes & KEGG \\
\hline Biological Process & BP \\
\hline Cellular component & CC \\
\hline Molecular function & MF \\
\hline Signaling Pathway & PA \\
\hline Differentially expressed genes & DEGs \\
\hline Differentially expressed miRNAs & DEmiRNAs \\
\hline Differentially expressed circRNA & DEcircRNA \\
\hline
\end{tabular}

\section{Declarations}

\subsection{Ethics approval and consent to participate}

There are no animals or clinical experiments in this study.

\subsection{Consent for publication}

It is not applicable.

\subsection{Funding}

No funding was received.

\subsection{Authors' contributions}

LNL and SYL collected the data, searched the literature and designed the study. LNL, SYL and FY conducted the experiments. LNL and FY analyzed the data, and interpreted the results. LNL drafted the 
manuscript. FY revised the manuscript. All authors read and approved the final manuscript.

\subsection{Acknowledgements}

We would like to thank Editage(www.editage.cn) for English language editing.

\subsection{Availability of data and materials}

The raw data supporting the conclusions of this article are available from the corresponding author on reasonable request. Our original data came from GEO database (Home - GEO - NCBI (nih.gov))

\subsection{Conflict of interest}

we have no financial and personal relationships with other people or organizations that can inappropriately influence our work, there is no professional or other personal interest of any nature or kind in any product, service and/or company that could be construed as influencing the position.

\section{References}

1. Barrett T, Wilhite SE, Ledoux P, Evangelista C, Kim IF, Tomashevsky M,Marshall KA, Phillippy KH, Sherman PM, Holko M, Yefanov A, Lee H, Zhang N,Robertson CL, Serova N, Davis S, Soboleva A. NCBI GEO: archive for functionalgenomics data sets-update. Nucleic Acids Res. 2013 Jan;41(Databaseissue):D991-5. doi: 10.1093/nar/gks1193. Epub 2012 Nov 27. PMID: 23193258;PMCID: PMC3531084.

2. Szklarczyk D, Morris JH, Cook H, Kuhn M, Wyder S, Simonovic M, Santos A,Doncheva NT,Roth A, Bork P, Jensen LJ, von Mering C. The STRING database in 2017: quality-controlled protein-protein association networks, made broadly accessible. Nucleic Acids Res. 2017 Jan 4;45(D1):D362-D368. doi:10.1093/nar/gkw937. Epub 2016 Oct 18. PMID: 27924014 ; PMCID: PMC5210637.

3. Shannon P, Markiel A, Ozier O, Baliga NS, Wang JT, Ramage D, Amin N, Schwikowski B, Ideker T. Cytoscape: a software environment for integrated models of biomolecular interaction networks. Genome Res. 2003 Nov;13(11):2498-504. doi:10.1101/gr.1239303. PMID: 14597658; PMCID: PMC403769.

4. Tang Z, Li C, Kang B, Gao G, Li C, Zhang Z. GEPIA: a web server for cancer and normal gene expression profiling and interactive analyses. Nucleic Acids Res. 2017 Jul 3;45(W1):W98-W102. doi: 10.1093/nar/gkx247. PMID: 28407145; PMCID:PMC5570223.

5. Chandrashekar DS, Bashel B, Balasubramanya SAH, Creighton CJ, Ponce-Rodriguez I, Chakravarthi BVSK, Varambally S. UALCAN: A Portal for Facilitating Tumor Subgroup Gene Expression and Survival Analyses. Neoplasia. 2017 Aug;19(8):649-658. doi: 10.1016/j.neo.2017.05.002. Epub 2017 Jul 18. PMID:28732212; PMCID: PMC5516091.

6. Li JH, Liu S, Zhou H, Qu LH, Yang JH. starBase v2.0: decoding miRNA-ceRNA, miRNA-ncRNA and protein-RNA interaction networks from large-scale CLIP-Seq data. Nucleic Acids Res. 
2014;42(Database issue):D92-D97. doi:10.1093/nar/gkt1248

7. Leonardi GC, Falzone L, Salemi R, et al. Cutaneous melanoma: From pathogenesis to therapy (Review). Int J Oncol. 2018;52(4):1071-1080. doi:10.3892/ijo.2018.4287

8. Schadendorf D, van Akkooi ACJ, Berking C, et al. Melanoma [published correction appears in Lancet. 2019 Feb 23;393(10173):746]. Lancet. 2018;392(10151):971-984. doi:10.1016/S01406736(18)31559-9

9. Darmawan CC, Jo G, Montenegro SE, et al. Early detection of acral melanoma: A review of clinical, dermoscopic, histopathologic, and molecular characteristics. J Am Acad Dermatol. 2019;81(3):805812. doi:10.1016/j.jaad.2019.01.081

10. Pavri SN, Clune J, Ariyan S, Narayan D. Malignant Melanoma: Beyond the Basics. Plast Reconstr Surg. 2016;138(2):330e-340e. doi:10.1097/PRS.0000000000002367

11. Cuevas LM, Daud Al. Immunotherapy for melanoma. Semin Cutan Med Surg. 2018;37(2):127-131. doi:10.12788/j.sder.2018.028

12. Franklin C, Livingstone E, Roesch A, Schilling B, Schadendorf D. Immunotherapy in melanoma: Recent advances and future directions. Eur J Surg Oncol. 2017;43(3):604-611. doi:10.1016/j.ejso.2016.07.145

13. Carpi S, Polini B, Fogli S, et al. Circulating microRNAs as biomarkers for early diagnosis of cutaneous melanoma. Expert Rev Mol Diagn. 2020;20(1):19-30. doi:10.1080/14737159.2020.1696194

14. Qi X, Zhang DH, Wu N, Xiao JH, Wang X, Ma W. ceRNA in cancer: possible functions and clinical implications. J Med Genet. 2015;52(10):710-718. doi:10.1136/jmedgenet-2015-103334

15. Huang X, Liu S, Wu L, Jiang M, Hou Y. High Throughput Single Cell RNA Sequencing, Bioinformatics Analysis and Applications. $A$

16. Jun L, Xuhong L, Hui L. Circ_SIPA1L1 Promotes Osteosarcoma Progression Via miR-379-5p/MAP3K9 Axis [published online ahead of print, 2020 Sep 4]. Cancer Biother Radiopharm. 2020;10.1089/cbr.2020.3891. doi:10.1089/cbr.2020.3891

17. Ge X, Li Z, Zhou Z, Xia Y, Bian M, Yu J. Circular RNA SIPA1L1 promotes osteogenesis via regulating the miR-617/Smad3 axis in dental pulp stem cells. Stem Cell Res Ther. 2020;11(1):364. Published 2020 Aug 24. doi:10.1186/s13287-020-01877-3

18. Li N, Miao Y, Shan Y, et al. MiR-106b and miR-93 regulate cell progression by suppression of PTEN via PI3K/Akt pathway in breast cancer. Cell Death Dis. 2017;8(5):e2796. Published 2017 May 18. doi:10.1038/cddis.2017.119

19. Drobna M, Szarzyńska B, Jaksik R, et al. hsa-miR-20b-5p and hsa-miR-363-3p Affect Expression of PTEN and BIM Tumor Suppressor Genes and Modulate Survival of T-ALL Cells In Vitro. Cells. 2020;9(5):1137. Published 2020 May 5. doi:10.3390/cells9051137

20. Chen J, O'Shea C, Fitzpatrick JE, Koster MI, Koch PJ. Loss of Desmocollin 3 in skin tumor development and progression. Mol Carcinog. 2012;51(7):535-545. doi:10.1002/mc.20818 
21. Cui T, Yang L, Ma Y, Petersen I, Chen Y. Desmocollin 3 has a tumor suppressive activity through inhibition of AKT pathway in colorectal cancer. Exp Cell Res. 2019;378(2):124-130. doi:10.1016/j.yexcr.2019.03.015

\section{Figures}

A

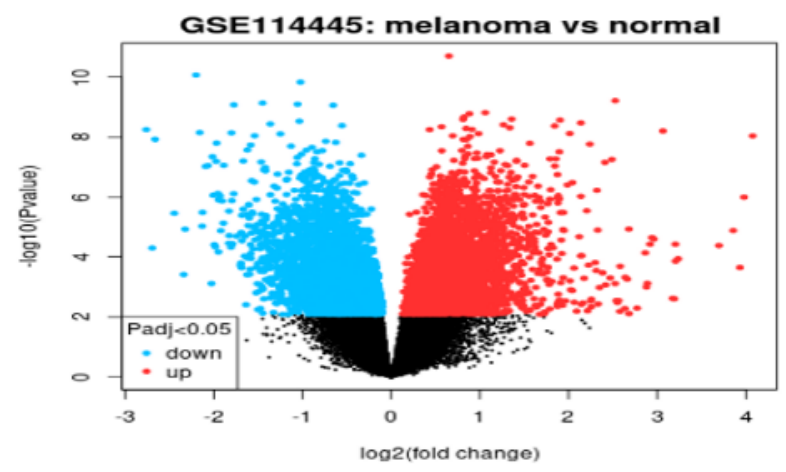

C

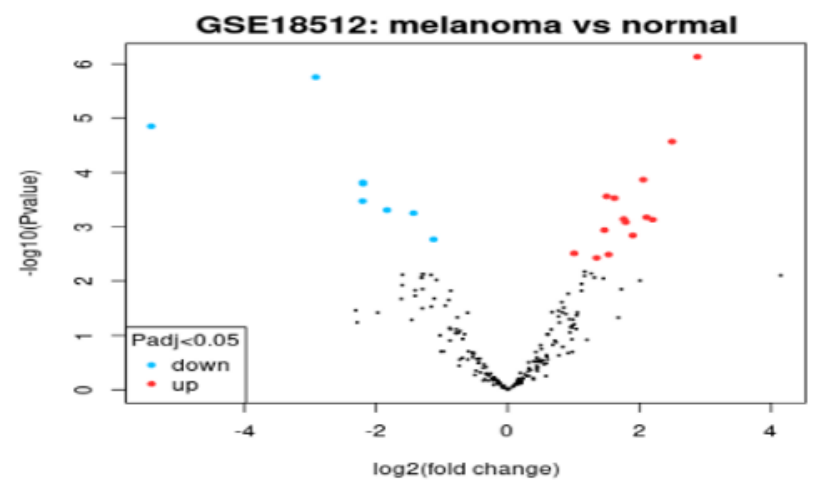

E

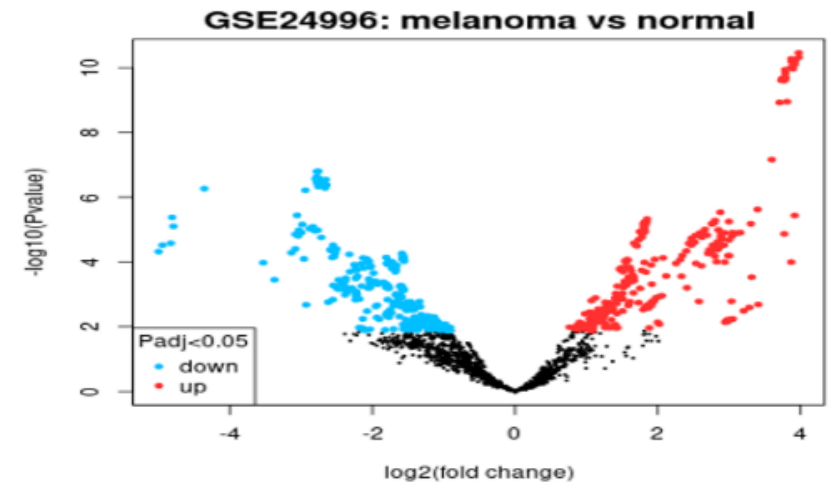

$\mathrm{B}$

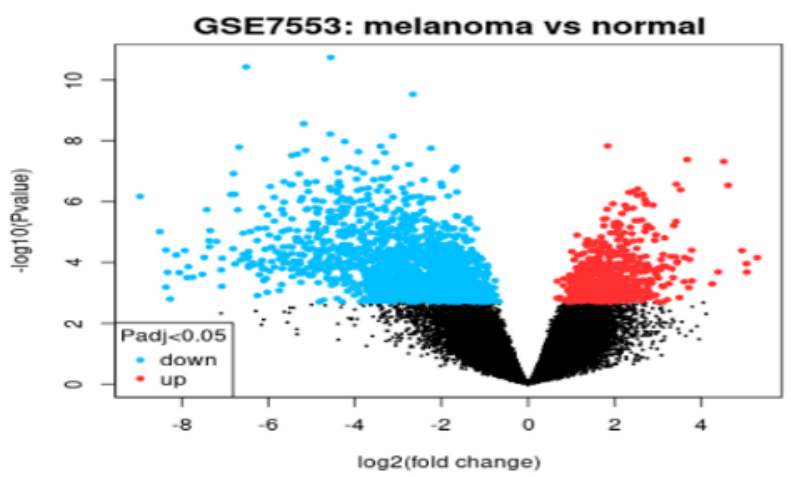

$\mathrm{D}$

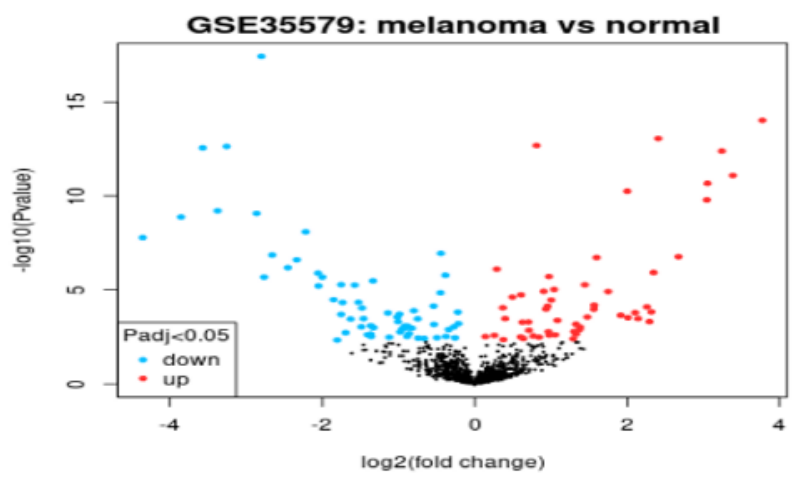

\section{Figure 1}

Volcanic maps of gene differential expressed.A Volcano Plot of GSE114445. B Volcano Plot of GSE7553. C Volcano Plot of GSE8512. D Volcano Plot of GSE35579. E Volcano Plot of GSE24996.Upregulated genes are marked in light red囚downregulated genes ars marked in light blue.(The differential expressed genes were selected with thresholds of $|\log F C|>1$ and $\mathrm{P}<0.05)$ 


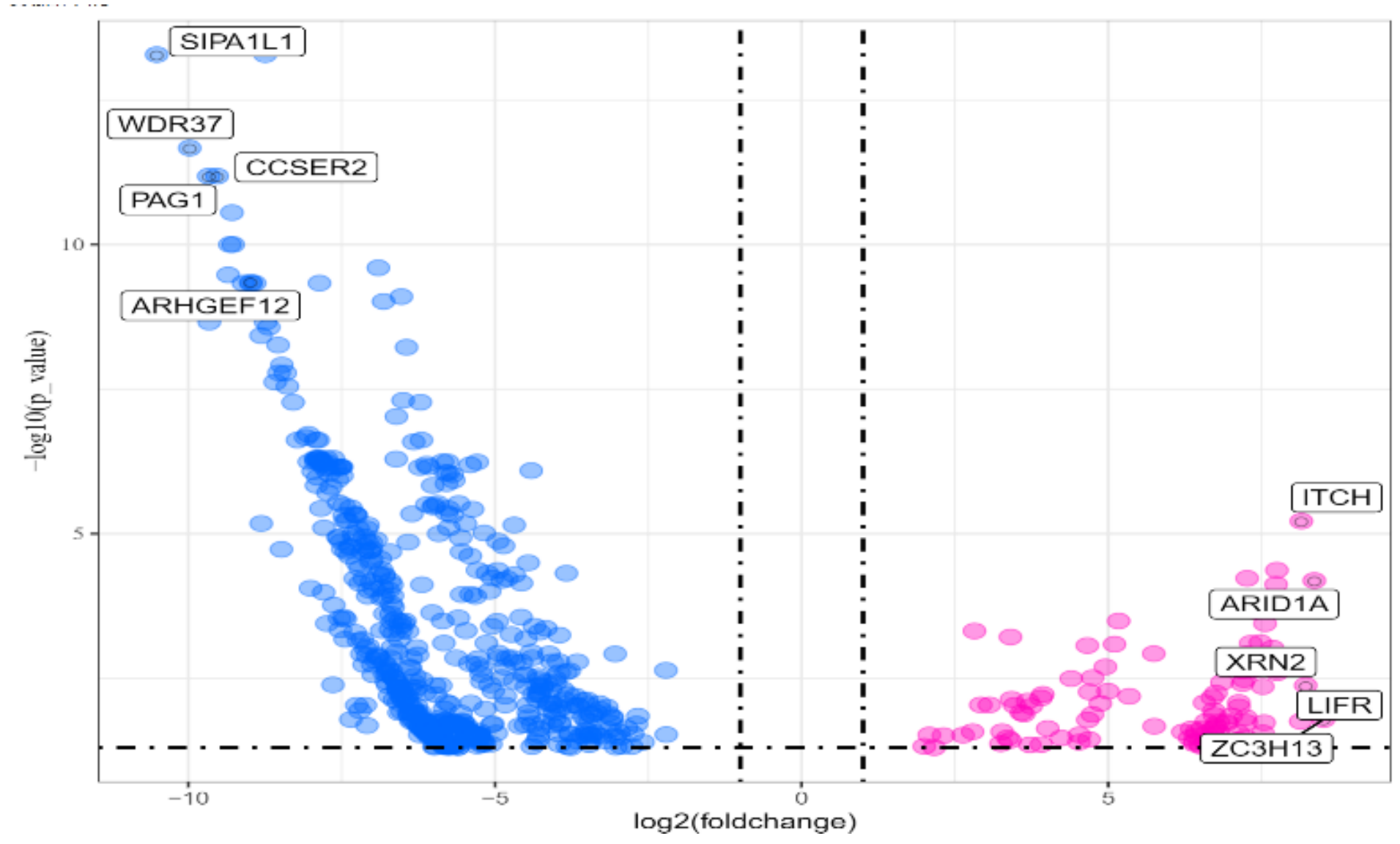

Figure 2

Volcano Plot of circRNA differential expressed.Upregulated genes are marked in light red囚downregulated genes ars marked in light blue.(The differential expressed genes were selected with thresholds of $\| \log F C \mid$ $>2$ and $\mathrm{P}<0.05)$ 
A

\section{GSE7553}

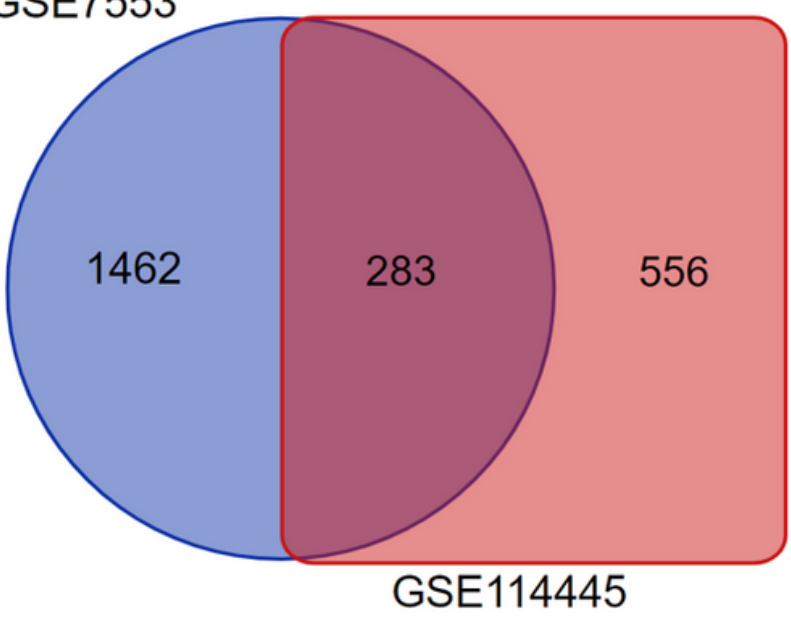

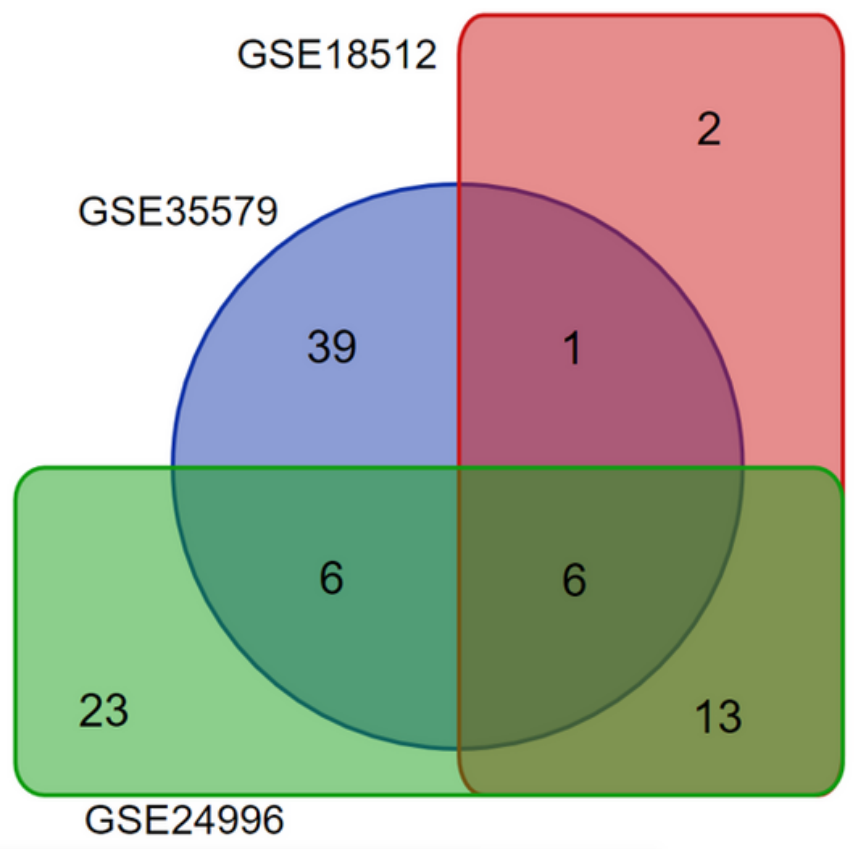

\section{Figure 3}

Venn of differentially expressed gene intersection. A the intersection of GSE114445 and GSE7553. B the intersection of GSE18512 GGSE35579 and GSE24996. (the genes contained in at least two data sets were selected as the target genes) 


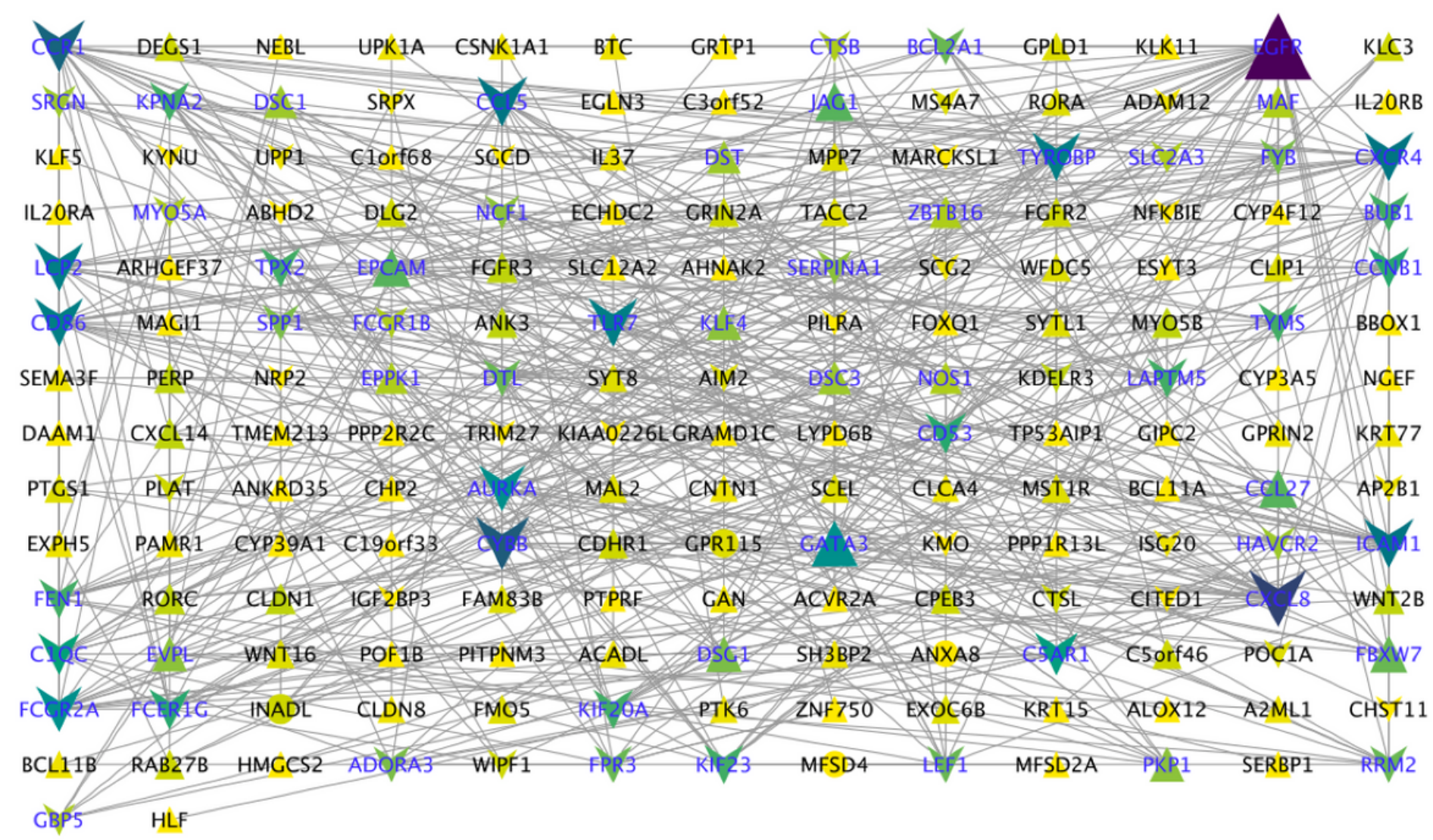

\section{Figure 4}

The PPI network of DEGs. Icons with different colors represent the degree value of the gene. The ligther the color, the smaller the degree value of the gene; Different icons represent the expression trend of the gene, the triangle the down-regulation of the gene expression in skcm, and the arrow symbol represents the up-regulation of the gene expression in skcm; The letters on the icon represest the name of the gene; The gene of purple letter is the hub gene of degree $>$ mean degree 


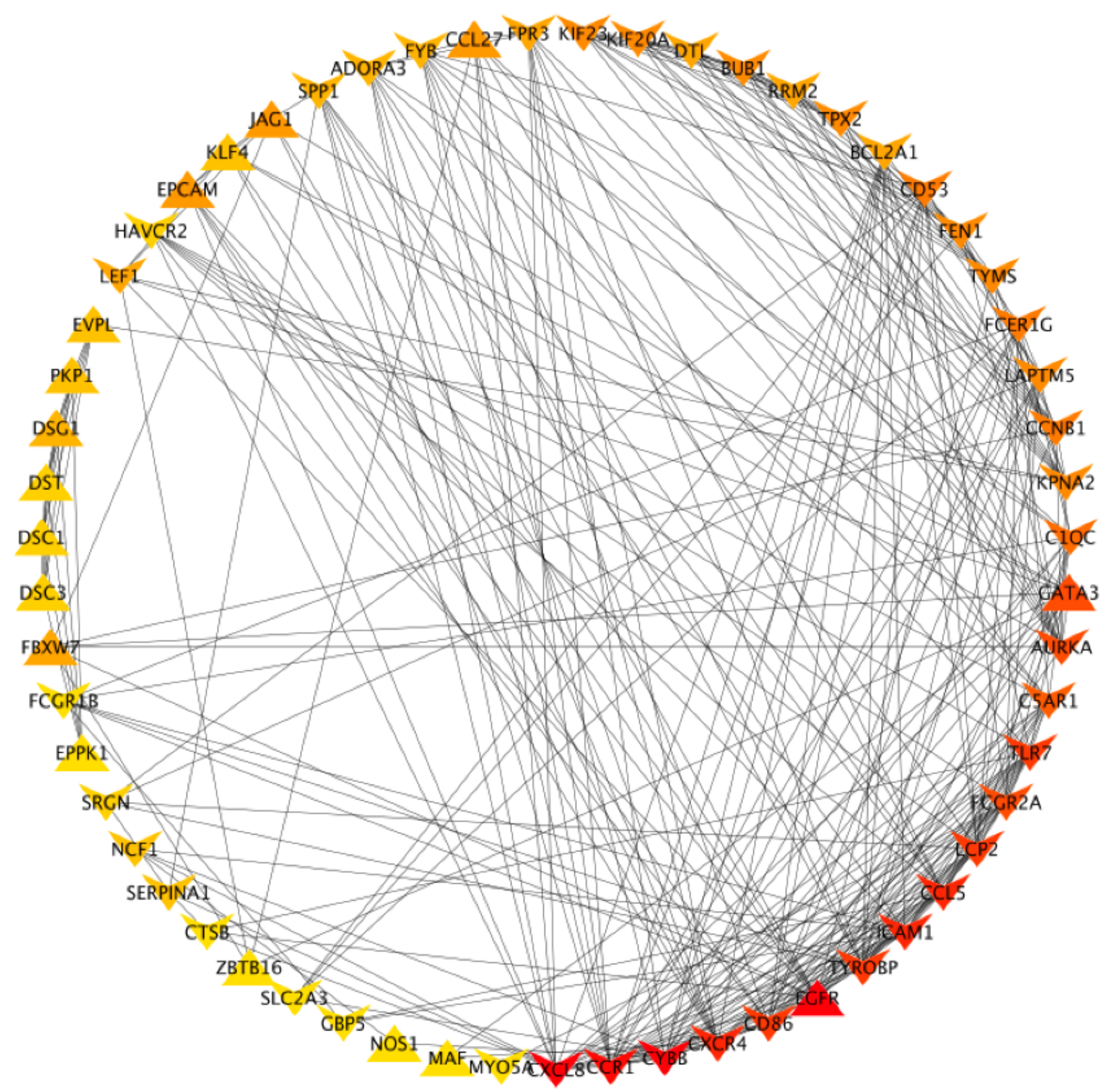

Figure 5

The subnetwork of Hub genes. Icons with different colors represent the degree value of the gene. The ligther the color, the smaller the degree value of the gene; Different icons represent the expression trend of the gene, the triangle the down-regulation of the gene expression in skcm, and the arrow symbol represents the up-regulation of the gene expression in skcm; The letters on the icon represest the name of the gene. 

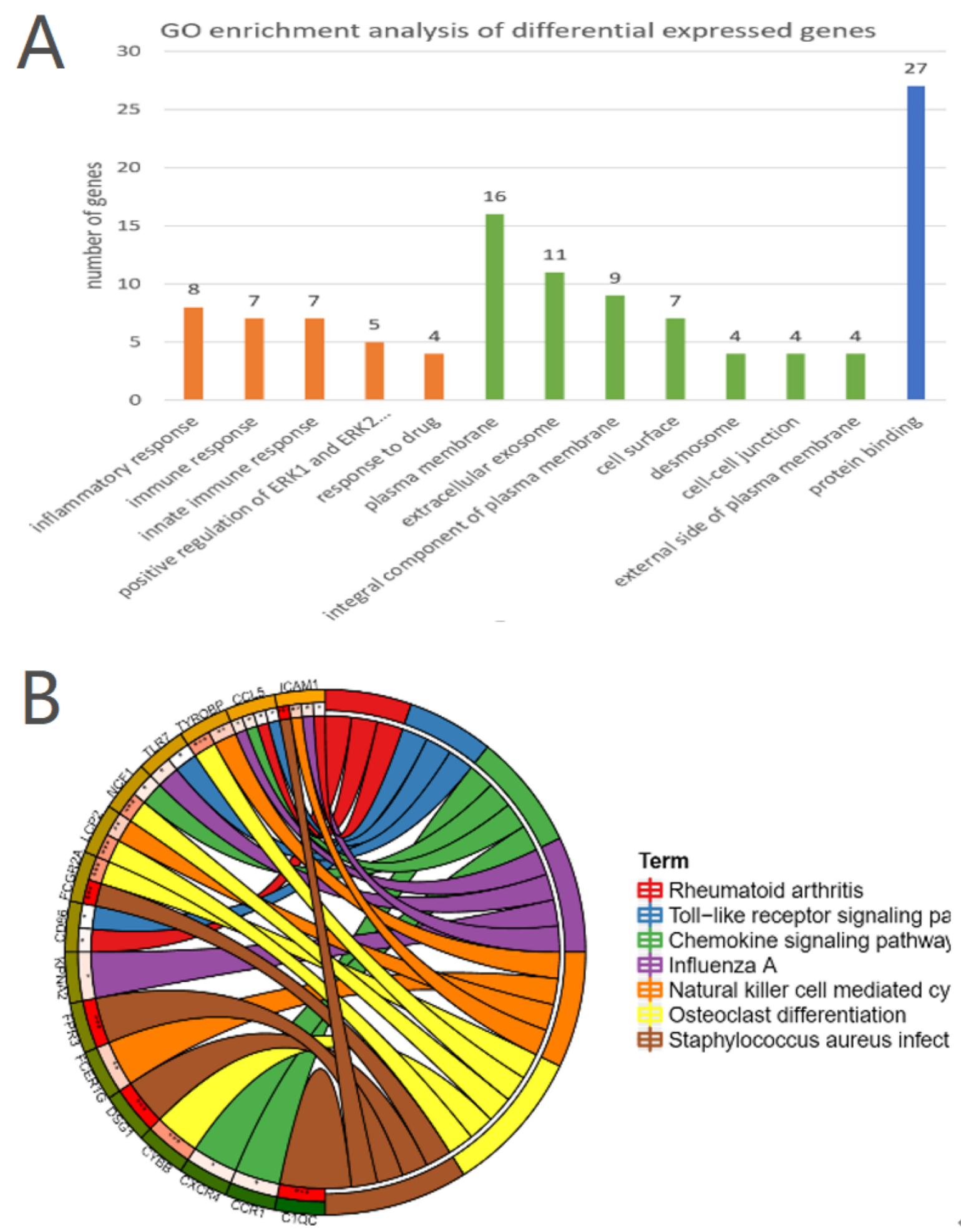

Term

申 Rheumatoid arthritis

申 Toll-like receptor signaling pa

申 Chemokine signaling pathwa) 申 Influenza A

申 Natural killer cell mediated cy

Osteoclast differentiation

申 Staphylococcus aureus infect

\section{Figure 6}

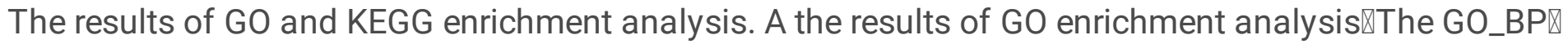
GO_MF and GO_CC were selected with thresholds of $\mathrm{P}<0.05$ and count $>3 \otimes \mathbb{B}$ B the results of KEGG enrichment analysis $₫$ The KEGG signaling pathways were selected with threshold of $P<0.05 \rrbracket$ 

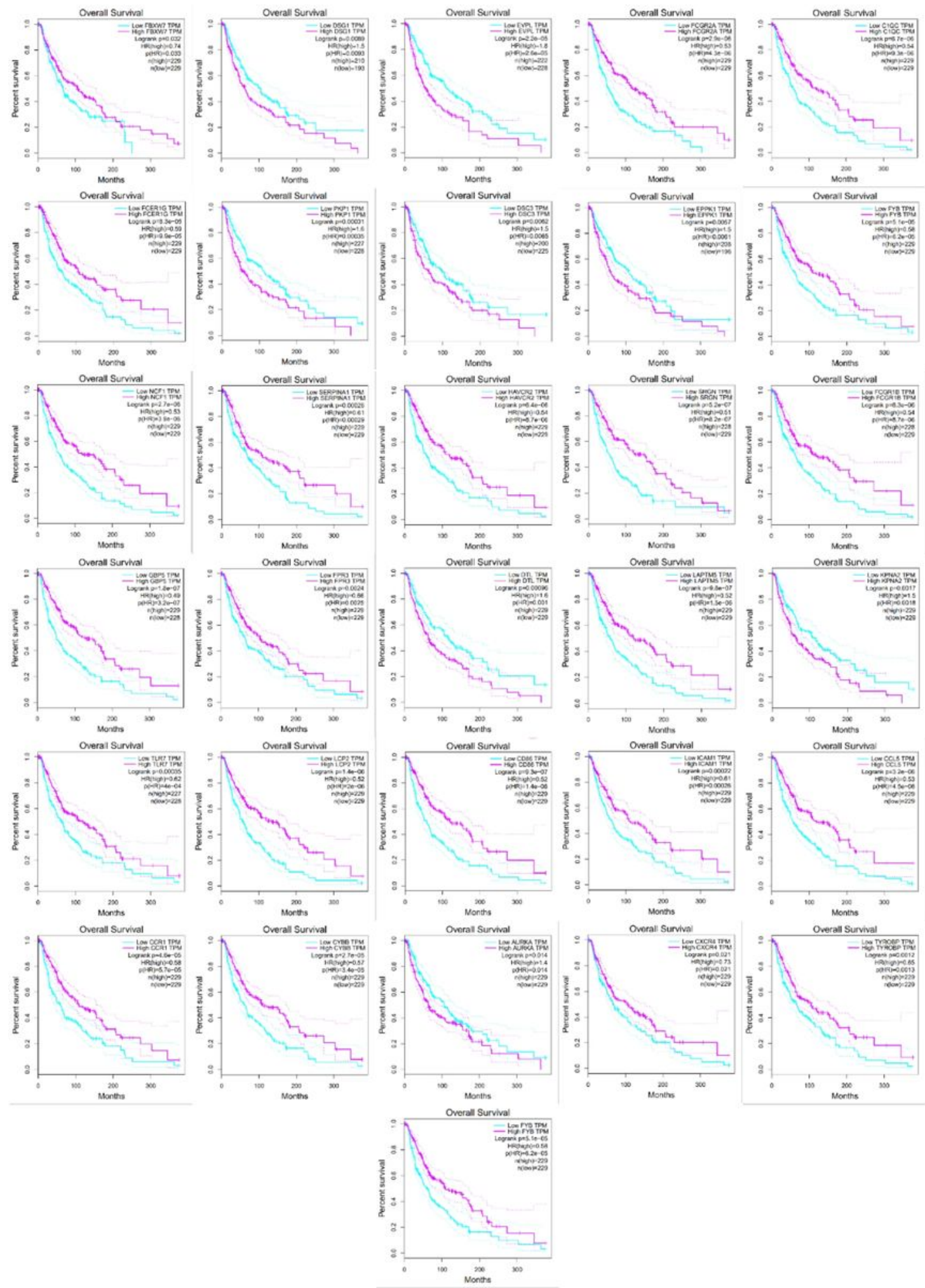

Figure 7

Correlation between mRNA and survival rate of SKCM. The mRNAs were selected with threshold of Logrank $\mathrm{P}<0.05$ 


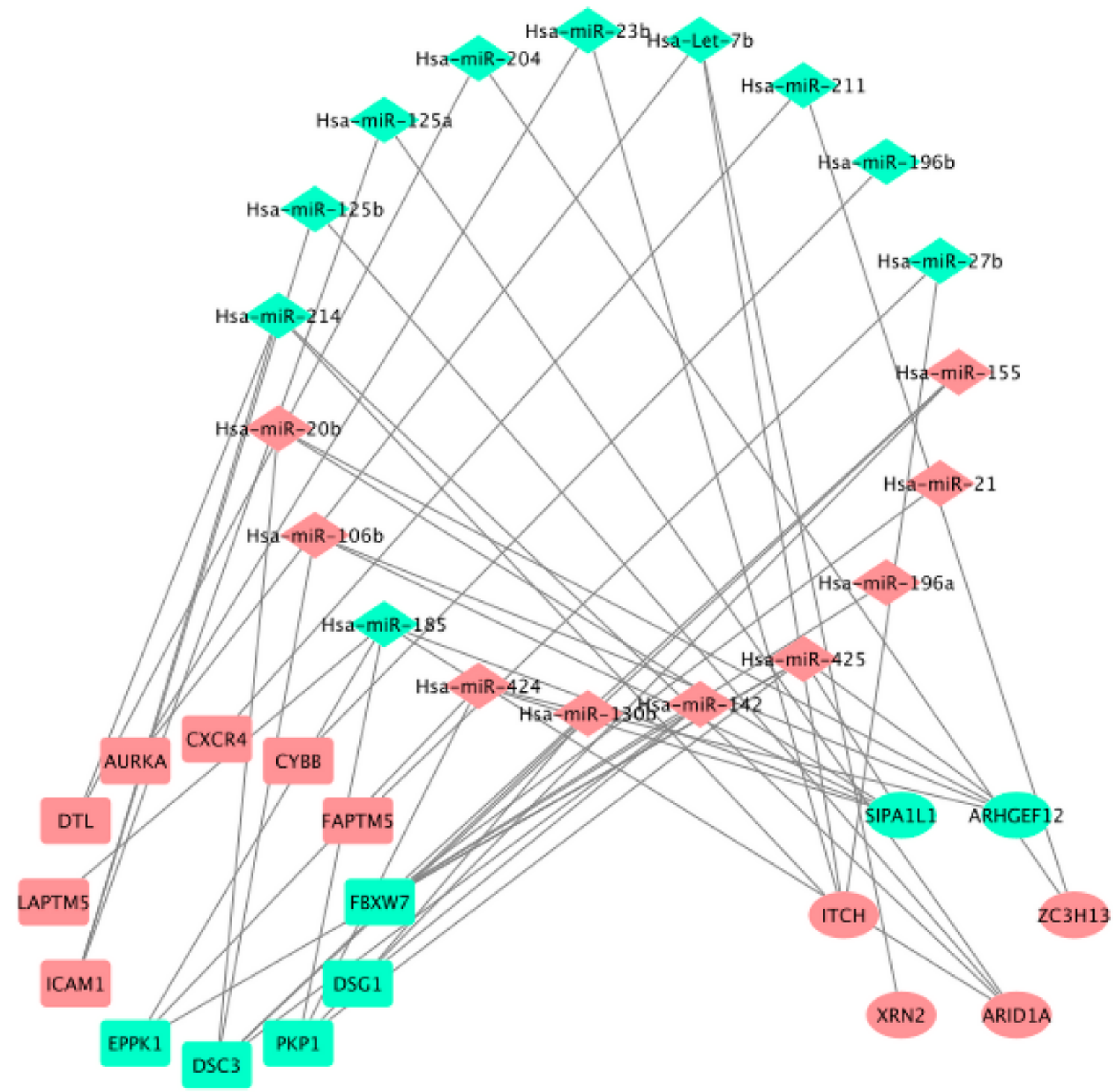

Figure 8

ceRNA Network Diagram. This figure clearly expressed the interaction among differentially expressed circRNA, microRNA and mRNA. The rectangular icon on the left represents differentially expressed mRNA; the oval icon on the right represesnts differentially expressed circRNA; the diamond icon in the middle represesnts the expressed microRNA. The color of the icon represents the expression trend of the gene in melanoma; Red is up-regulated and green is down-regulated. 


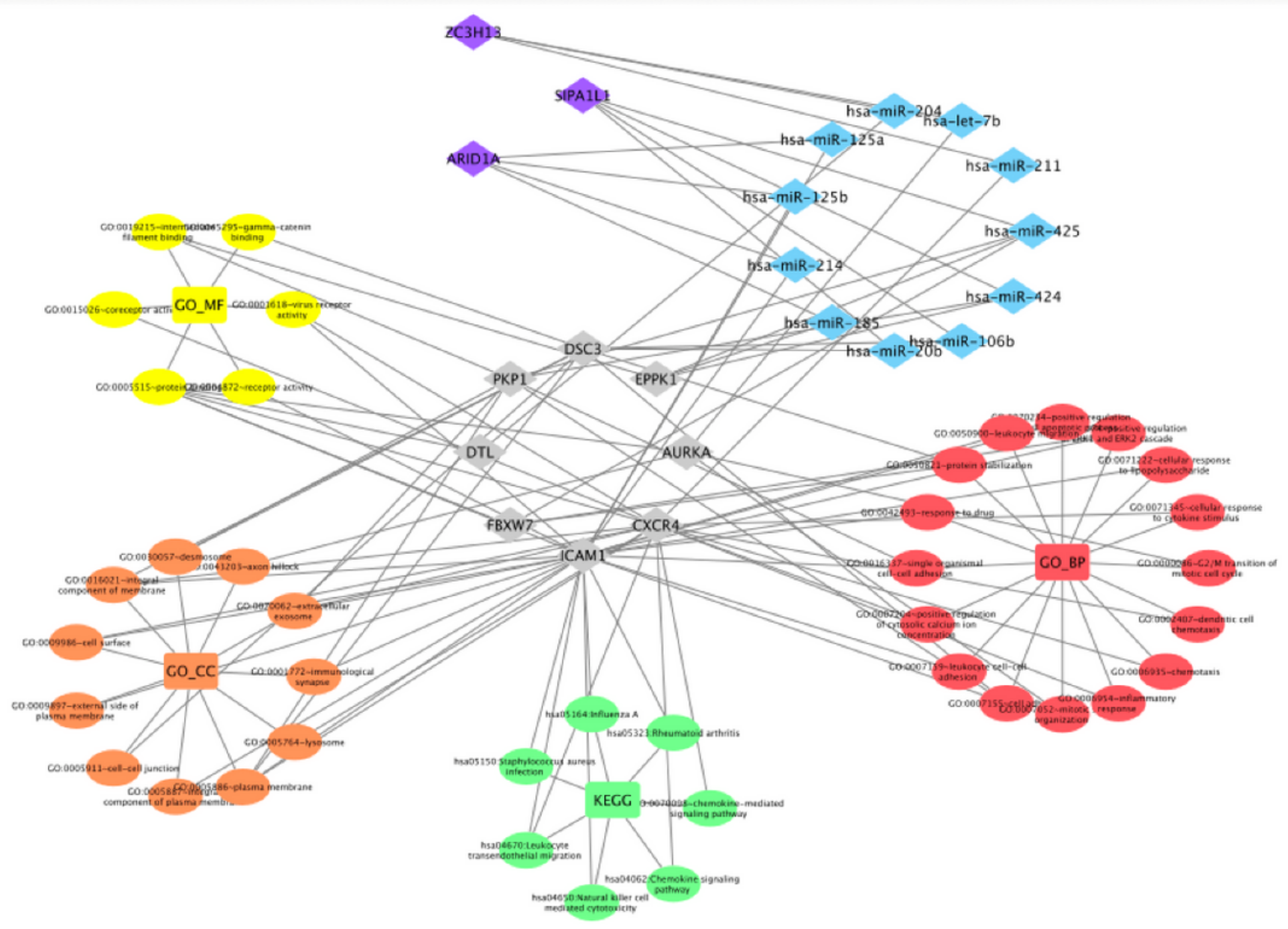

Figure 9

Genes-GO/KEGG enrichiment network. Gray icon represents mRNA, blue icon represesnts miRNA, purple icon represents circRNA; the red icon represents biological effect(GO_BP) enrichment analysis results, the orange icon represents cell localization(GO_CC) enrichment analysis results, the yellow icon represents molecular function(GO_MF) enrichment analysis results. 


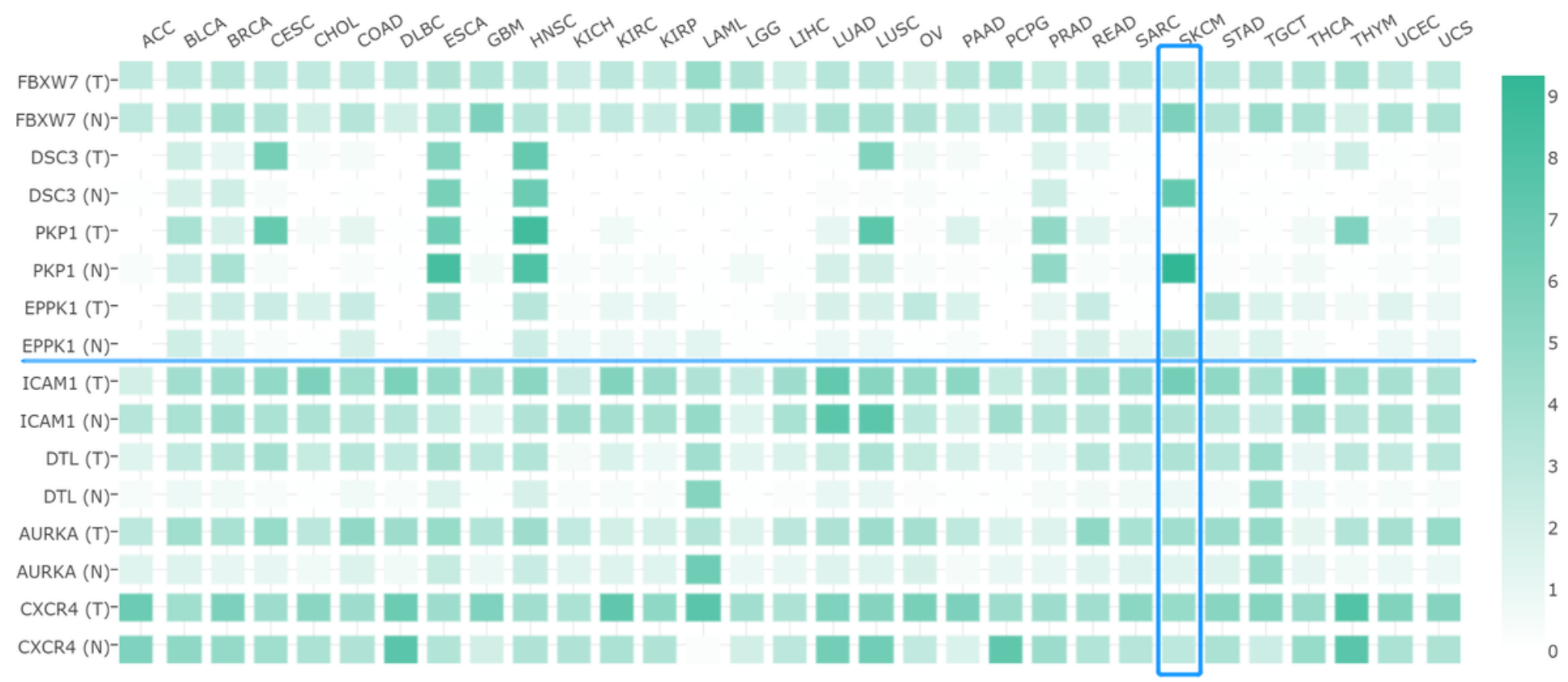

\section{Figure 10}

Target mRNA differentially expressed analysis in Pan-cancer. The darker the color of the box, the higher the expression of the gene in the corresponding tissues. It can be seen from the figure that DSC3, PKP1 and EPPK1 have the most specific expression in skin melanoma. 

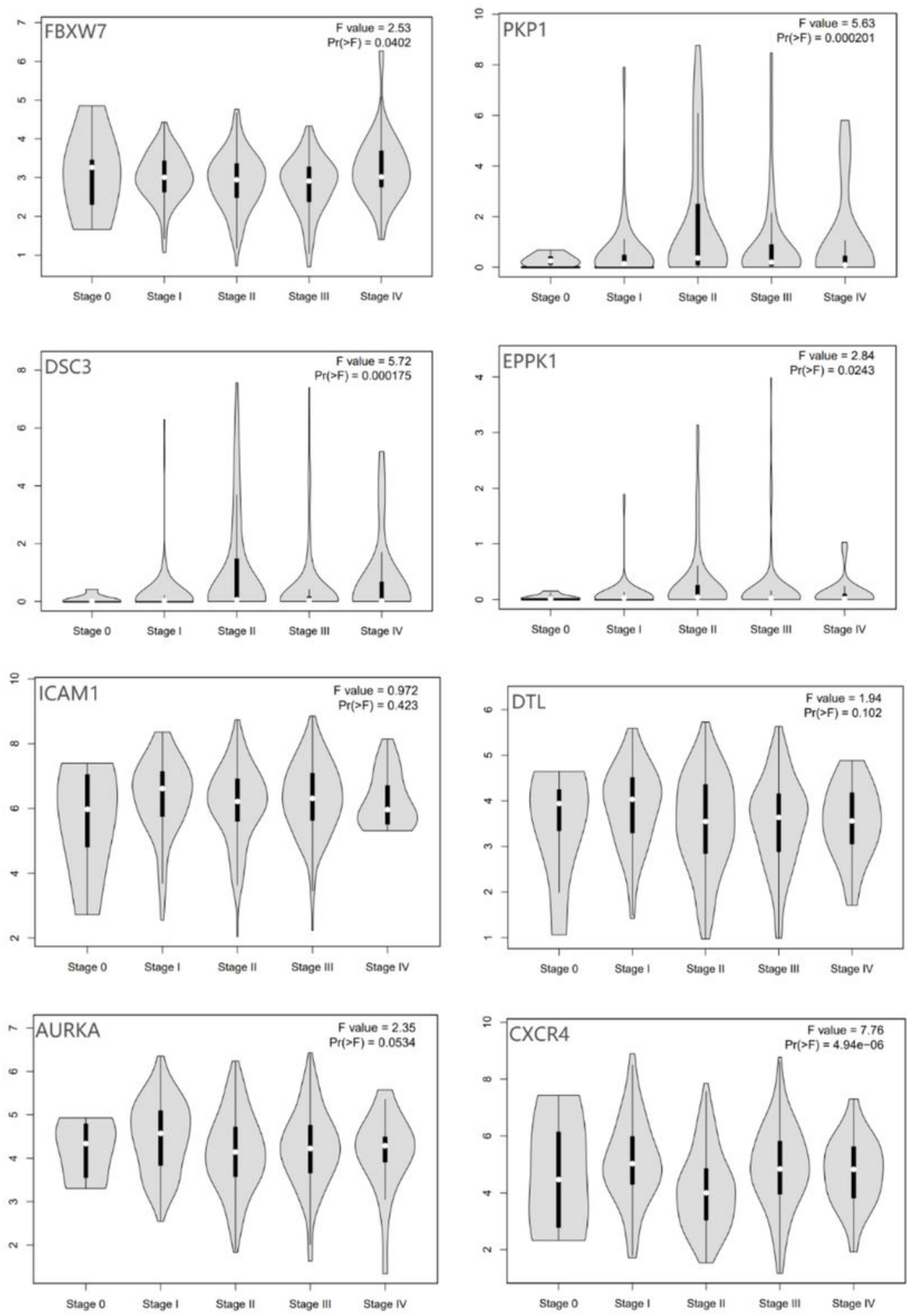

Figure 11

The expression of mRNA in different stages of skin cutaneous melanoma. It can be seen from the figure that FBXW7, PKP1, DSC3, EPPK1 and CXCR4 have related to the stages of skin cutaneous melanoma $\triangle T$ The genes were selected with threshold of $P<0.05 \rrbracket$. 

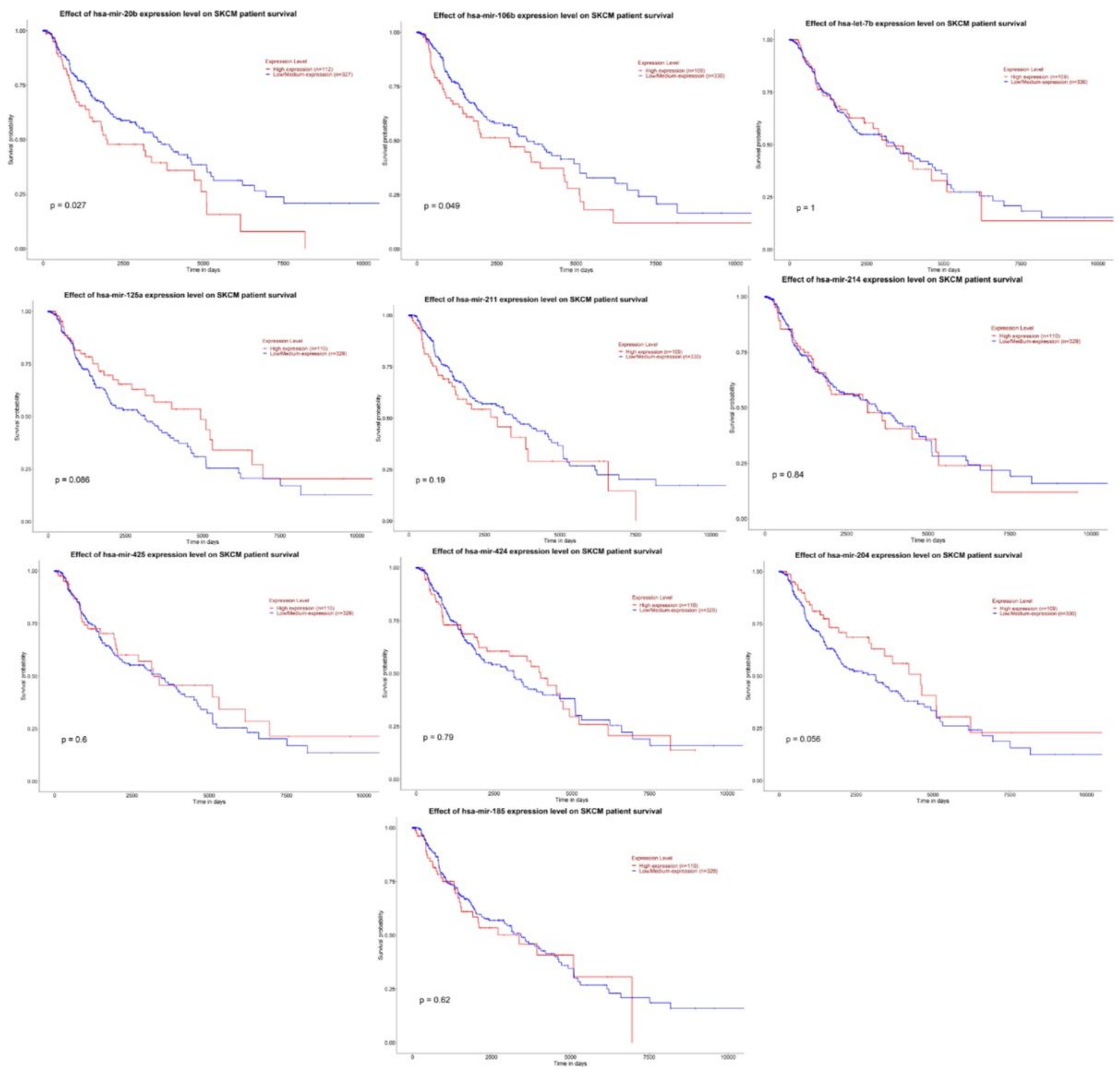

Figure 12

Survival analysis of miRNA in skin cutaneous melanoma. It can be seen from the figure that has-miR$106 \mathrm{~b}$ and has-miR-20b have related to th e survival rate of skin cutaneous melanoma冈The miRNAs were selected with threshold of $\mathrm{P}<0.05$. 


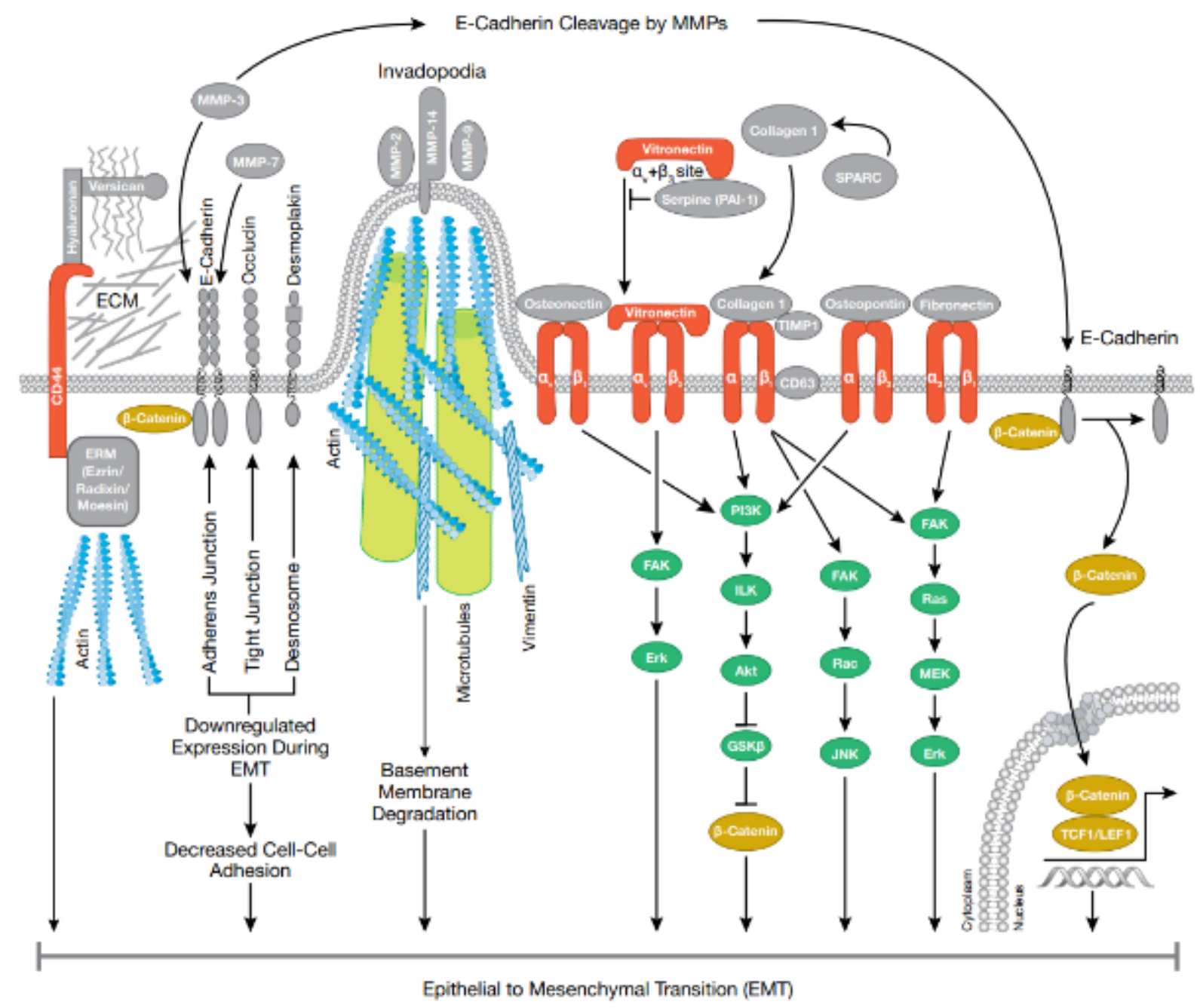

Figure 13

Cell adhesion signaling pathway. E-cadherin is the main cadherin located in epithelial cells. The loss of DSC3 leads to reduced E-cadherin expression in epithelial cells, lack of cell adhesion, and increase in cell motility, which may further induce epithelial-mesenchymal transition (EMT) of tumour cells through multiple signalling pathways. 